\title{
Análisis del Comportamiento de la Siniestralidad por Enfermedades Catastróficas en una Empresa Promotora de Salud-Colombia
}

Luis Hernando Nieto-Enciso

Médico, Especialista en Gerencia de la Salud Pública. Hospital Chapinero E.S.E., División de Salud Pública. Secretaría de Salud de Bogotá. E-mail: hernandonieto@yahoo.com

Recibido 22 Abril 2005/Enviado para Modificación 25 Abril 2005/Aceptado 11 Agosto 2005

\section{RESUMEN}

Objetivos Identificar las tendencias en el comportamiento de la siniestralidad por enfermedades catastróficas y los determinantes de las variaciones presentadas por patología y régimen en afiliados a SaludCoop, Empresa Promotora de Salud.

Métodos Se realizó un análisis de tendencias en razón de uso y la relación costo/ingresos de la Unidad de Pago por Capitación, frente a estudios actuariales de referencia.

Resultados Para el periodo 1997-1999 en el régimen contributivo, la atención en Unidad de Cuidado Intensivo (adultos) y la Cirugía Cardiovascular constituyeron las patologías que generan mayor costo de atención; en el régimen subsidiado corresponden a servicios de quimioterapia y Unidad de Cuidado Intensivo Neonatal (URN).

Conclusiones Las tendencias de la siniestralidad estudiada evidencian la necesidad de implementar modelos de atención que fortalezcan la gestión de riesgos, intervenciones prioritarias en el área preventiva y la vigilancia epidemiológica de los eventos de alto costo.

Palabras Clave: Costos y análisis de costo, costos de la atención en salud, seguro de salud, fondos de seguro (fuente: DeCS, BIREME).

ABSTRACT

Analysing catastrophic illness patterns in a Health Promoting Company in Colombia

Objective Identifying trends in catastrophic illness patterns as well as determinant factors of variation due to pathology and type of mandatory contri- 
bution regime in people affiliated to SaludCoop (a health promoting company forming part of Colombian's health and social security system).

Methods Trends were analysed based on why the service was used and cost/income ratio obtained by per capita payment unit compared to actuarial reference studies.

Results Intensive care unit (ICU) attention for adults and cardiovascular surgery were the pathologies leading to greater health costs 1997-1999 in the mandatory employees' insurance contribution regime. Chemotherapy and neonatal intensive care led to the greatest costs in the mandatory subsidised health insurance regime.

Conclusions The catastrophic illness patterns studied revealed the need for establishing attention models aimed at improving risk management, priority intervention in the field of prevention and epidemiological surveillance of high cost illnesses.

Key Words: Catastrophic illness, cost and cost analysis, health care cost, health insurance, insurance pool (source: MeSH, NLM).

$\mathrm{E}$ l presente artículo pretende analizar las tendencias y variaciones en el comportamiento de las enfermedades catastróficas y las diferencias existentes entre los parámetros utilizados para el cálculo de la Unidad de Pago por capitación frente al comportamiento de la siniestralidad encontrado para estos eventos en una Empresa Promotora de Salud.

Este análisis resulta especialmente importante en cuanto la "desviación" frente a patrones de demanda esperada afecta la viabilidad y equilibrio financiero del Sistema de Seguridad Social y puede ocasionar: deficiencias en la calidad de la atención al superar la capacidad instalada de los prestadores/aseguradores, saturar los servicios hospitalarios o "absorber" recursos inicialmente asignados a otros niveles de atención.

Si bien "ha sido y es todavía difícil predecir la estructura y el comportamiento de la demanda de servicios de salud para el tratamiento de enfermedades de alto costo, necesarios para efectos de planeación prospectiva, presupuestación del gasto en salud y para identificar mecanismos que mejoren la eficiencia del Sistema” (1), una caracterización de las dinámicas y comportamientos de las enfermedades de alto costo permitirán profundizar en el estudio de factores de riesgo, incidir en el diseño de políticas de formación del recurso humano en salud, disponer de criterios de priorización en la asignación de recursos para investigación y evaluación de nuevas tecnologías. . 
En el desarrollo del Sistema de Seguridad Social en Salud Colombiano, se evidencian fenómenos de concentración y/o selección de riesgos entre administradores de planes de beneficios, siendo los eventos de alto costo los que mayor efecto pueden generar sobre la viabilidad financiera y la calidad en la prestación de servicios de algunas aseguradoras.

En este contexto, en diciembre de 2001, se reconoció por parte del Consejo Nacional de Seguridad Social en Salud (CNSSS) la desviación del perfil epidemiológico para patologías de alto costo (2), iniciando a partir de allí el diseño de una serie de mecanismos encaminados a generar la redistribución de riesgos, en cumplimiento del marco legal vigente que establece “...corresponde al CNSSS definir las medidas necesarias para evitar la selección adversa de usuarios por parte de las Entidades Promotoras de Salud y una distribución inequitativa de los costos de la atención de los distintos tipos de riesgo" (3). Así, en enero de 2003, el CNSSS estableció una política de atención integral de patologías de alto costo (4) que incluyo cuatro componentes:

- La implementación de mecanismos de redistribución del riesgo

- El control de la selección del riesgo

- Ajustes al Modelo de atención y

- Procedimientos de Vigilancia epidemiológica para eventos de cuarto nivel.

En el marco de esta política, se ordenó una redistribución de afiliados (con patologías de VIH-SIDA o Insuficiencia Renal Crónica) entre aseguradoras por una única vez, a llevarse a cabo en junio de 2003, con el propósito de que cada Empresa Promotora de Salud (EPS) y Administradoras de Régimen Subsidiado (ARS) tuvieran un número proporcional de casos en función del numero total de afiliados, procurando homogenizar el nivel de riesgo entre aseguradores.

\section{ALGUNAS PRECISIONES CONCEPTUALES}

Las enfermedades "Catastróficas o de Alto Costo" se definen en el Manual de Actividades, Procedimientos e Intervenciones del Plan Obligatorio de Salud (5), como aquellas que representan una alta complejidad técnica en su manejo, alto costo, baja ocurrencia y bajo "costo - efectividad” en su tratamiento. Corresponden según la normatividad a: tratamiento con radioterapia y quimioterapia para el cáncer, diálisis para insuficiencia renal crónica, transplantes (renal, de corazón, de medula ósea y de cornea), tratamiento 
para el SIDA y sus complicaciones, tratamiento quirúrgico para enfermedades del corazón y del sistema nervioso central, tratamiento quirúrgico para enfermedades de origen genético o congénito, tratamiento medico quirúrgico para el trauma mayor, terapia en unidad de cuidados intensivos, reemplazos articulares y atención del Gran Quemado-en el régimen subsidiado (6).

Condiciones de cobertura y aseguramiento

Las patologías de alto costo son atendidas en el Sistema de Seguridad Social Colombiano bajo un esquema diferencial entre el régimen contributivo y Subsidiado. En el régimen subsidiado se ofrece una cobertura plena desde el momento mismo de afiliación mientras que en el régimen contributivo se obliga al cumplimiento de un "Periodo mínimo de cotización" correspondiente a 100 semanas, de las cuales por lo menos 26 deben haber sido cotizadas en el último año (7) o la cobertura parcial del evento en proporción al tiempo cotizado al Sistema. En ambos regímenes estos eventos están excluidos del pago de copagos o cuotas moderadoras (8).

Reaseguros en eventos de alto costo

Dentro del esquema de aseguramiento en salud, la normatividad obliga a que los Administradores de planes de beneficios tomen un "reaseguro" con el propósito de cubrir las posibles desviaciones en el costo de los eventos presentados.

Aunque existen diversos modelos de reaseguros, en principio en todos los esquemas se mantiene la figura del pago de "Primas" con coberturas delimitadas y esquemas diferenciales para aplicación de deducibles, mediante la cual las EPS/ARS transfieren parte del riesgo al reasegurador. Los efectos financieros y la decisión de optar por uno u otro de los esquemas de deducibles dependen de la siniestralidad presentada frente a modelos probabilísticos predeterminados.

\section{Conceptos sobre siniestralidad}

Se entiende por "Siniestralidad" el conjunto de eventos presentados y objeto de cobertura por un seguro. Se diferencia del concepto de "riesgo", pues mientras el primero expresa una certeza el segundo se relaciona con la probabilidad de ocurrencia de un evento.

La siniestralidad es resultado de tres variables: la frecuencia de uso, la razón de uso y los costos promedio de atención de un evento. La razón de uso 
corresponde al número de atenciones ocasionadas por el manejo de una patología, en un periodo de tiempo dado con relaciona una población de referencia y la frecuencia de uso establece el promedio de demanda de un servicio por una persona en un periodo de tiempo determinado.

En el marco de la conceptualización expuesta, el presente estudio abordó los comportamientos de la siniestralidad en una población asegurada, en el intento de comprender las fluctuaciones y desviaciones de la demanda de servicios.

\section{METODOLOGÍA}

El análisis de las tendencias de la siniestralidad por eventos de alto costo se abordó tomando como unidad de análisis los siniestros ocurridos en el período 1997 - 1999 en una Empresa Promotora de Salud - SaludCoop EPS - e identifica las variaciones en la razón de uso entre régimen de afiliación, el comportamiento de los costos de atención por tipo de patología y los factores determinantes de la variabilidad presentada.

Se tomó como fuente de información la base de datos de "Eventos de alto costo" presentados y reportados en la EPS SaludCoop suministrada por la Dirección Nacional de Investigación y Estadística de dicha EPS.

La misma comprende en el régimen contributivo 9989 casos cuyos costos de atención ascendieron a 33387 millones de pesos y en el régimen subsidiado a 1249 casos por un valor de 3274 millones de pesos. Sobre la base de datos se realizó un proceso de depuración con dos propósitos: garantizar el cumplimiento de los criterios de inclusión establecidos en la Ley y realizar una reclasificación de eventos en aquellas situaciones donde confluyeran dos tipos de servicios de cuarto nivel asociados por alguna patología catastrófica de base (por ejemplo Unidad de Cuidado Intensivo derivado de una neurocirugía). En estos casos se reclasificaron los eventos asignándole la causa primaria de atención.

Con la información disponible se calculó el número total de eventos y el costo total de atención (valorado a tarifas del Instituto de Seguros Sociales vigentes al momento de atención) por tipo de patología catastrófica. Posteriormente se calculó la razón de uso, como una medida del riesgo potencial de enfermar y el porcentaje de gasto de atención de cada evento frente a los ingresos operacionales (porcentaje de la Unidad de pago por capitaciónUPC) 
A partir de la información procesada se desarrolló un análisis comparativo de la siniestralidad esperada vs. encontrada, (tomando como referencia los estudios actuariales definidos por el Gobierno Nacional al momento de definición de la UPC), y la generación de hipótesis explicativas sobre las variaciones y diferencias encontradas.

\section{RESULTADOS}

Comportamiento de los costos de atención y siniestralidad. SaludCoop EPS. 1997-1999

El análisis del comportamiento de costos frente a ingresos operacionales, reflejó sensibles diferencias en las tendencias presentadas, entre la población del régimen contributivo y la población afiliada al subsidiado y por tipo de patología estudiada.

La Tabla 1 presenta los resultados al comparar los costos de atención por eventos de alto costo vs. ingresos operacionales; de la misma se deduce, que los costos de atención son superiores en el régimen contributivo con relación al subsidiado. No obstante, en el periodo estudiado en el régimen contributivo las fluctuaciones observadas fueron moderadas; en contraposición, en el régimen subsidiado se presentó un notable incremento (del $105 \%$ ) en los costos de atención por enfermedades catastróficas.

Tabla 1. Relación costo de eventos catastróficos/Ingresos operacionales. SaludCoop EPS.

\begin{tabular}{|c|c|c|}
\hline Periodo & Contributivo (\%) & Subsidiado (\%) \\
\hline 1997 & 6,6 & 2,2 \\
\hline 1998 & 7,8 & 2,1 \\
\hline 1999 & 7,2 & 5,0 \\
\hline
\end{tabular}

SaludCoop EPS. 2.000 .

Esta situación podría derivar de los siguientes fenómenos, (que se mantendrán como hipótesis de trabajo a verificar durante el análisis):

- Mejor conocimiento de derechos y coberturas por parte de los actores del Sistema en el régimen contributivo, facilitando procesos de remisión a niveles superiores y adquisición progresiva de los mismos en el régimen subsidiado. 
- Fortalecimiento progresivo de las redes de servicios y de la capacidad de acceso a los mismos para usuarios del régimen subsidiado contribuyendo asi al incremento progresivo de costos.

- Dificultades en accesibilidad u oportunidad a servicios curativos de baja y mediana complejidad derivando en complicaciones y uso de servicios de alta complejidad.

- Procesos de afiliación selectivos en el regimen subsidiado por parte de los Entes Territoriales en los que se dio prioridad a pacientes previamente diagnosticados con enfermedades catastróficas originando un incremento sustancial en los costos de atención.

Para facilitar el análisis de los fenómenos presentados se realizo inicialmente una discriminación de costos por patología, comparando los patrones de cada régimen de afiliación, en el propósito de entender las tendencias encontradas (Figuras 1 y 2 ).

Una primera revisión de la información graficada muestra que mientras en el régimen contributivo la atención en Unidad de Cuidado Intensivo (adultos) y la Cirugía Cardiovascular constituyen los rubros que generan mayor costo de atención, en el régimen subsidiado corresponden a servicios de quimioterapia y Unidad de Cuidado Intensivo Neonatal (URN)

El análisis comparativo de las Figuras 1 y 2, permite identificar además:

- un comportamiento decreciente de los costos por cirugía cardiovascular en el régimen contributivo lo cual indicaría la resolución progresiva de la carga de enfermedad acumulada por esta patología y la dilución del riesgo por el volumen alcanzado de usuarios.

- un aumento progresivo en los costos (equivalente a un $100 \%$ ), por servicios de quimioterapia en el régimen subsidiado (mas acentuado que en el régimen contributivo) lo cual fortalecería la hipótesis de afiliación selectiva.

- Y en el régimen subsidiado una tendencia creciente, en los costos generados por servicios de unidad de cuidado intensivo neonatal (aumentaron en un $1000 \%$ ), consecuencia de serias dificultades en la calidad de servicios materno perinatales (crisis hospitalaria), implementación progresiva de nuevas tecnologías y progresivo mejoramiento en la accesibilidad a este tipo de servicios.

- Para el evento "Diálisis Renal” se observa un incremento progresivo tanto en el régimen contributivo (del $130 \%$ ) como en el régimen 
subsidiado (del 600 \%) que seria consecuencia de las cinco hipótesis planteadas .

Figura 1. Comportamiento del costo frente al ingreso por tipo de patología catastrófica. SaludCoop EPS. Régimen Contributivo. Colombia 1997-2000

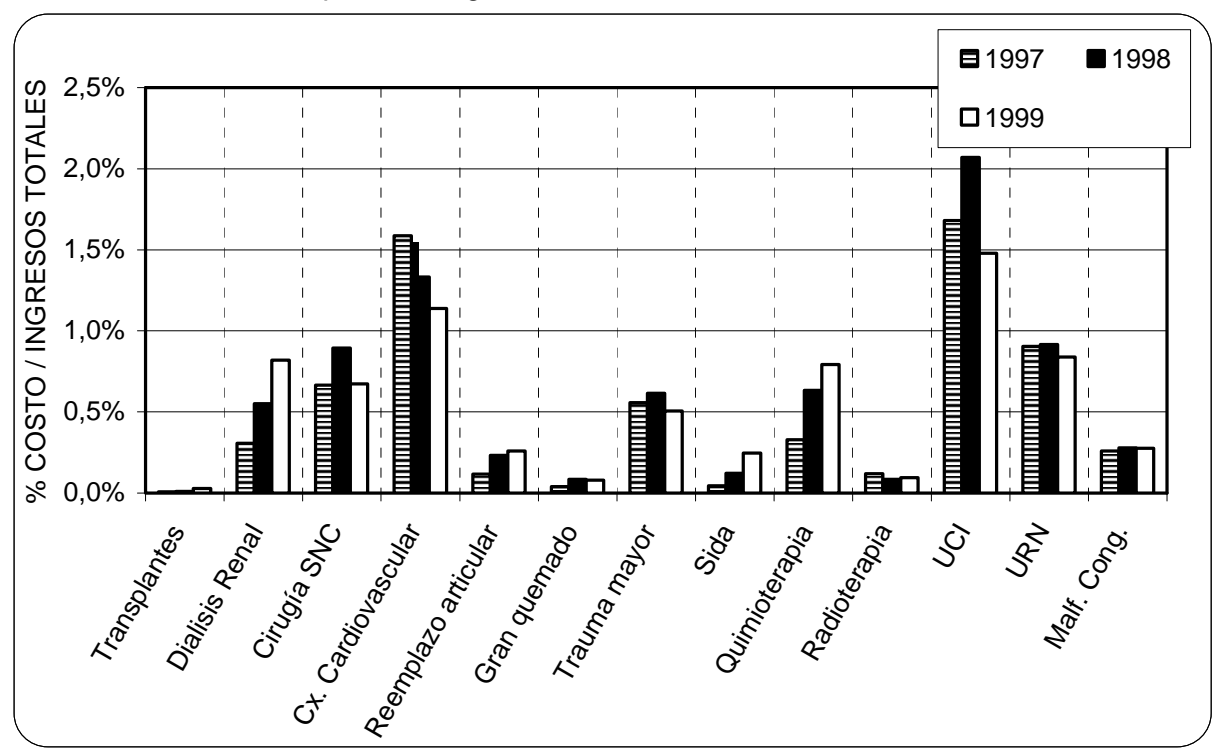

Fuente: Dirección Nacional de Investigación y Estadística. SaludCoop EPS. 2000.

Para efectos del análisis se compararon adicionalmente las razones de uso esperadas ( según estudios actuariales que sirvieron de supuestos para el cálculo de la UPC) frente a las encontradas para cada régimen en el año 1999.

Los hallazgos sobre siniestralidad esperada contra la encontrada se presentan en la Tabla 2. Como se puede apreciar para todas las patologías cubiertas se presenta una brecha significativa entre las frecuencias de uso proyectadas en los estudios actuariales frente a la demanda atendida en la población objeto (principalmente para transplantes, diálisis renal y unidad de cuidados intensivos)

Igualmente debe resaltarse la ausencia de estadísticas y frecuencias esperadas para los eventos de trauma mayor, quimioterapia/radioterapia, Unidad de cuidado intensivo neonatal y tratamiento quirúrgico de malformaciones congénitas. La ausencia de tal información obligaría a reevaluar la suficiencia de la UPC como consecuencia de información que haya sido precisada durante el desarrollo del Sistema de Seguridad Social. 
Figura 2. Comportamiento del costo frente al ingreso por tipo de patología catastrófica. Saludcoop EPS. Régimen Subsidiado. Colombia 1997-2000

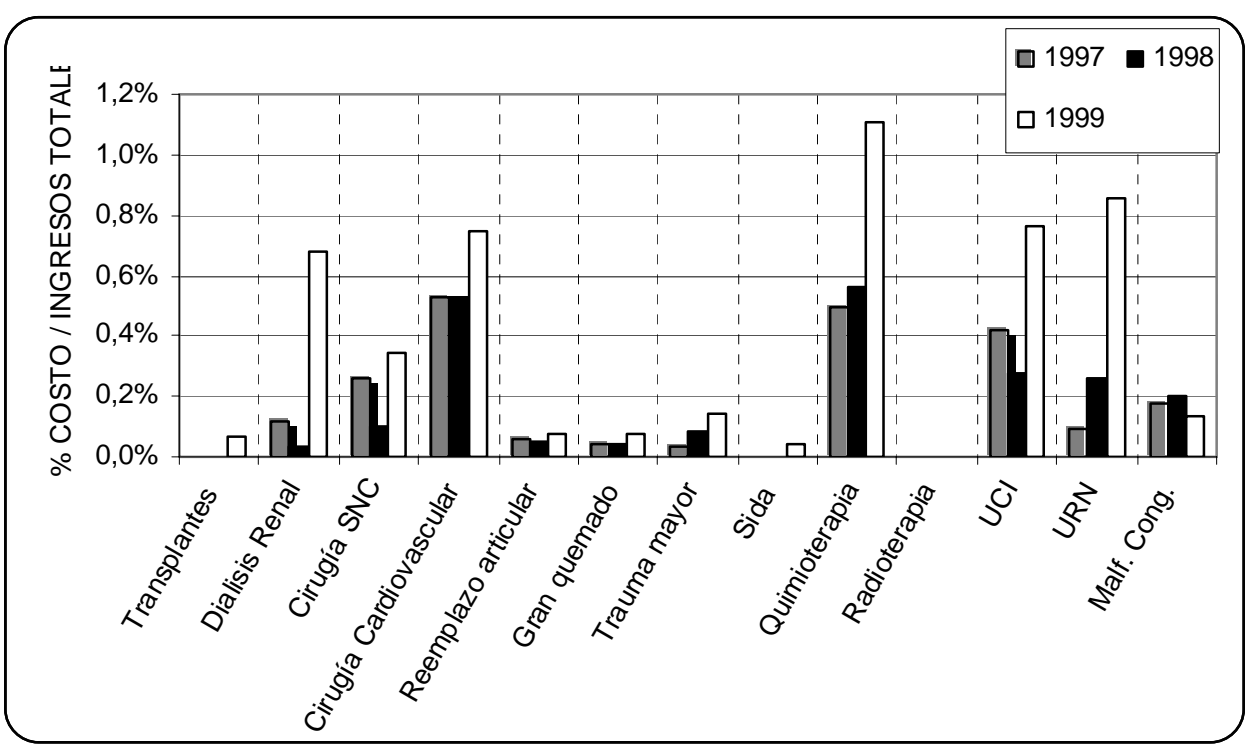

Fuente: Dirección Nacional de Investigación y Estadística. SaludCoop EPS. 2000.

Tabla 2. Razón de Uso esperada vs. presentada, por tipo de patología catastrófica, por régimen. SaludCoop EPS. Colombia 1999.

\begin{tabular}{|c|c|c|c|}
\hline \multirow[t]{2}{*}{ Patología } & \multirow{2}{*}{$\begin{array}{l}\text { Razón de uso } \\
\text { Esperada }\end{array}$} & \multicolumn{2}{|c|}{$\begin{array}{c}\text { Razón de uso } \\
\text { Observada (1999) }\end{array}$} \\
\hline & & Contributivo & Subsidiado \\
\hline Transplantes & 0,00032 & 0,00001 & 0,00002 \\
\hline Diálisis Renal & 0,00050 & 0,00045 & 0,00022 \\
\hline Cirugía SNC & 0,00028 & 0,00034 & 0,00010 \\
\hline Cirugía Cardiovascular & 0,00019 & 0,00025 & 0,00016 \\
\hline Reemplazo articular & 0,00020 & 0,00009 & 0,00002 \\
\hline Gran quemado & 0,00003 & 0,00003 & 0,00002 \\
\hline Trauma mayor & N.D. & 0,00063 & 0,00013 \\
\hline SIDA & 0,00010 & 0,00037 & 0,00004 \\
\hline Quimioterapia & N.D. & 0,00097 & 0,00073 \\
\hline Radioterapia & N.D. & 0,00005 & 0,00000 \\
\hline $\mathrm{UCl}$ & 0,00356 & 0,00026 & 0,00011 \\
\hline URN & N.D. & 0,00027 & 0,00023 \\
\hline Malformaciones Congénitas & N.D. & 0,00038 & 0,00010 \\
\hline
\end{tabular}

Fuente: Dirección Nacional de Investigación y Estadística. SaludCoop EPS. 2000 
Por otra parte, debe resaltarse las diferencias en las frecuencias de uso entre regímenes, encontrando en principio que para la mayoría de patologías estas son mayores en el régimen contributivo.

Lo anterior sería resultado de los siguientes factores:

- La población afiliada objeto de análisis del régimen contributivo era predominantemente urbana y la del régimen subsidiado rural. Esta situación influye en la accesibilidad a servicios, disponibilidad de medios diagnósticos y de tratamiento.

- Capacidad económica de los afiliados que repercute en la posibilidad o no de desplazarse a ciudades metropolitanas donde se dispone del recurso humano y tecnológico necesario para el tratamiento.

- La situación de crisis presentada en la red pública especializada en el período estudiado. Esta constituye en algunos departamentos la única opción de acceso a población del régimen subsidiado y como resultado de la situación descrita se presentaban cierres temporales de servicios, dificultades para garantizar oportunidad y continuidad en los servicios para pacientes de cuarto nivel.

En el caso de VIH/SIDA se observa, además, una diferencia importante en las frecuencias de uso, (10 veces mayor en el contributivo) lo cual podría estar relacionado con el soporte social brindado por familiares y allegados para procurar condiciones de atención propias del régimen contributivo, modalidades de asociación entre pacientes que evidenciaban mayor capacidad de presión social y exigibilidad de derechos y percepción de mayor capacidad de atención, idoneidad técnico científica o respuesta efectiva a necesidades por parte de algunas EPS del régimen contributivo.

\section{DISCUSION}

El estudio presenta una metodología novedosa de análisis de la siniestralidad por enfermedades catastróficas, al articular tres componentes: aspectos epidemiológicos descriptivos, un análisis retrospectivo comparativo del comportamiento de costos en poblaciones con condiciones socioeconómicas diferenciadas y una contextualizacion socio histórica explicativa, pretendiendo un acercamiento a algunos de los determinantes de las variaciones observadas.

Los hallazgos demuestran variaciones importantes entre los supuestos disponibles al momento del diseño de la Unidad de Pago por Capitación con 
relación a las frecuencias de uso y costos observados en una población de referencia. En consecuencia, seria recomendable desarrollar investigaciones futuras que permitan un análisis integral y universal del comportamiento de las enfermedades de alto costo en Colombia en el propósito de fortalecer líneas de investigación clínico epidemiológicas, fortalecer los programas de formación del recurso humano en salud y ajustar la Unidad de Pago por Capitación para evitar riesgos financieros en el Sistema de Seguridad Social.

A pesar de que uno de los fundamentos a la Reforma de Seguridad Social en Salud en Colombia se basa en la implementación de un modelo preventivo, las tendencias y comportamientos observados en el presente estudio indican que es preciso fortalecer y repensar los modelos de gestión de riesgos y las políticas de promoción y prevención en salud a fin de mejorar la efectividad de las acciones en salud •

Agradecimientos. A los Doctores Carlos Palacino Antia, Maria Fernanda Isaacs, Edgar Mendoza, Nelcy Paredes y a la profesional en estadística Diela Moreno, por sus comentarios, aportes conceptuales y en el análisis de datos y a Alba María Blanco por el estímulo y generosidad en el tiempo dedicado a la elaboración a este estudio.

\section{REFERENCIAS}

1. Toro W. Modelo de simulación prospectiva de servicios de salud para enfermedades de alto costo: aplicación para una entidad promotora de salud colombiana. Pág. 1-18. [Internet] Disponible en: www.funsalud.org.mx/redjlb/premio04.hmt\#walter. Consultado mayo de 2005.

2. Consejo Nacional de Seguridad Social en Salud. República de Colombia Acuerdo 217 del 30 de diciembre de 2001. Por el cual se establece un mecanismo para el reconocimiento de la desviación del perfil epidemiológico de la atención en salud del régimen contributivo. Bogotá.

3. Ministerio de Salud Pública. República de Colombia. Ley 100 de 1993, articulo 172, numeral 9.

4. Consejo Nacional de Seguridad Social en Salud. República de Colombia Acuerdo 245 del 30 de enero de 2003. Por el cual se establece la política de atención integral de patologías de alto costo, para los regimenes del contributivo y subsidiado del SGSSS. Bogotá

5. Ministerio de Salud Pública. República de Colombia. Resolución 5261 del 5 de agosto de 1994. Manual de Actividades, Procedimientos e Intervenciones del Plan Obligatorio de Salud. Bogotá.

6. Consejo Nacional de Seguridad Social en Salud. República de Colombia Acuerdo 72 de 1997. Por medio del cual se define el Plan de Beneficios del Régimen Subsidiado. Bogotá

7. Ministerio de Salud Pública. República de Colombia Decreto 806 de 1998. Por el cual se reglamenta la afiliación al Régimen de Seguridad Social en Salud 
304 REVISTA DE SALUD PÚBLICA • Volumen 7 (3), Noviembre 2005

y la prestación de los beneficios del servicio público esencial de Seguridad Social en Salud y como servicio de interés general, en todo el territorio nacional. Bogotá.

8. Consejo Nacional de Seguridad Social en Salud. República de Colombia. Acuerdos 30 de 1995 y 260 de 2004. Por medio del cual se define el régimen de copagos y cuotas moderadoras dentro del Sistema General de Seguridad Social en Salud. Bogotá 